\title{
Aglaonema tassai (Araceae; Tribe: Areae) - sp. nov. from Arunachal Pradesh, Northeast India
}

\author{
Hui Tag ${ }^{1}$ and Atek Nangkar \\ Plant Systematic and Ethnobotanical Research Laboratory, Department of Botany, Rajiv Gandhi \\ University, Rono Hills, Doimukh-791112, Arunachal Pradesh, India \\ ${ }^{1}$ Corresponding author; e-mail: huitag2008rgu@gmail.com
}

[Received 01.11.2018; Revised 29.11.2018; Accepted 08.12.2018; Published 31.12.2018]

\begin{abstract}
Abstruct
Aglaonema tassai H.Tag \& A.Nangkar, a new species of Aglaonema Schott (Araceae) recognized from Rottung, East Siang District of Arunachal Pradesh in Northeast India, has been described and illustrated with labelled diagram and color photographs including that of Holotype. This new species resembles Aglaonema hookerianum Schott, A. modestum Schott ex Engl. and $A$. simplex (Blume) Blume but significantly differ in stem, leaves, inflorescence, cataphylls, spathe, spadix and fruit characters. Main distinguishing morphological characters and key to the locally available species are provided for its easy identification.
\end{abstract}

Key words: Aglaonema tassai, New species, Endemic, Arunachal Pradesh, India

\section{INTRODUCTION}

The genus Aglaonema (Araceae; Tribe: Areae) was established by Schott in 1829 and has 32 accepted species with 99 synonyms as recorded in version 1.3 of www.theplantlist.org. This genus inhabits humid and heavily shaded forests of southeastern Asia, northeastern India, southern China, Indonesia, Malaysia and New Guinea (Li \& Boyce 2010; Hay 1998). Nicolson (1969) from Smithsonian Institute was the first to revise the genus Aglaonema Schott of Southeast Asia, and has reported the existence of 76 binomials of which 21 are accepted. These plants are primarily native to Southeast Asian countries. Karthikeyan et al. (1989) have mentioned five species of Aglaonema from India whereas J.D. Hooker (1893) reported the existence of Aglaonema hookerianum Schott (from Khasia Hills, Cachar and Chittagong) and Aglaonema clarkei Hook.f. (from Chittagong). However, A. clarkei is now treated as a synonym of $A$. hookerianum.

The Indian state of Arunachal Pradesh is situated on a part of the Eastern Himalayan chain of mountains endowed with rich tropical to temperate broadleaved and moist forest types those favoured the luxuriant growth of several Aglaonema species. However, majority of Arunachal Himalayan regions are still remains unexplored due to remoteness and inaccessible mountain terrains. While exploring the aroid flora of the state, the authors collected one species of Aglaonema Schott from Rottung (altitude 400 - 700 m) in East Siang District of Arunachal Pradesh in the year 2014. During further intensive botanical field survey such a plant was not observed outside of the East Siang range. The perusal of literature and critical study of the specimen revealed that it is a new species of the genus Aglaonema that is closely related to $A$. hookerianum Schott, A. modestum Schott ex Engl. and A. simplex (Blume) Blume. This population is now named as Aglaonema tassai, sp. nov., and is presented here along with suitable description, illustrations and coloured photographs. 


\section{Taxonomic treatment:}

Aglaonema tassai H.Tag \& A.Nangkar, sp. nov. [PLATE - I]

Perennial herbs, evergreen with dark green stem; basal stem, terete, decumbent; branches sub-erect, terete, $21-40 \mathrm{~cm}$ high, $1.5-2.6 \mathrm{~cm}$ in diameter; internodes $1-2 \mathrm{~cm}$ long, rooting from places just below the nodes. Cataphylls $3-5$, early caducous, slightly yellow, white to green, oblong to lanceolate, obtuse to \pm acute, $3-4 \mathrm{~cm}$ long, $1-1.5 \mathrm{~cm}$ wide. Leaves $1-4$ (usually 2), not crowded at stem apex; petioles $13-18 \mathrm{~cm}$ long, $1-2 \mathrm{~cm}$ wide, dark green, base sheathing. Lamina elliptic to ovate-oblong, $22-31 \mathrm{~cm}$ x $13-15$ $\mathrm{cm}$, entire, caudate-acuminate, base tunicate-rounded, green, non-variegated, lateral veins $7-20$, running parallel to each other. Inflorescence generally 1, rarely 2 at the same time from leaf axils. Peduncle short, major part $(80-90 \%)$ remain covered with cataphylls (sometime almost up to spathe base), $5 \mathrm{~cm}$ long, $0.5 \mathrm{~cm}$ wide, green, erect, much shorter than petiole. Spathe canoe-shaped, light yellow or white to green, $4-5 \mathrm{~cm}$ long, $2.5 \mathrm{~cm}$ wide, obovate, entire, acute - acuminate and curved to $80-90,1-2 \mathrm{~cm}$ part of spathe in lower and upper extremes never open. Spadix sessile, $3.1 \mathrm{~cm}$ long, shorter then spathe, about $1.2 \mathrm{~cm}$ short of spathe apex; Female part free, not adnate to spathe, $1 \mathrm{~cm}$ long, 0.8 $\mathrm{cm}$ wide; ovary green, slightly globose - oval, 1-loculed, 1-ovuled; stigma sessile, white, circular, concave; Male part $2.1 \mathrm{~cm}$ long, $0.6 \mathrm{~cm}$ wide, whitish, terete tip rounded, deciduous; stamens 2, united; anthers with porous dehiscence. Berries not much crowded, ovoid, 0.4 $-0.6 \mathrm{~cm}$ long and $0.3 \mathrm{~cm}$ thick, green, red when ripe, 1 -seeded.

The basic taxonomic characters of new species Aglaonema tassai closely resembles with A. hookerianum Schott, A. modestum Schott ex Engl. and A. simplex (Blume) Blume in having terete stem, visible nodes and internode, terete spadix, and absence of staminodes and sterile zone, but significantly differ from them by having only $1-4$ leaves which are not crowded at the tip, and absence of a stipe. Aglaonema tassai differ with Aglaonema simplex by having erect but much shorter and decumbent stem, leaves $2-4$, not crowded at stem apex, lateral veins 6 - 20 each side, spathe canoe-shaped, short peduncle, male zone, terete with rounded apex, ovary oval, seed oval; and differ with Aglaonema modestum by having less or none crowded leaf at stem apex, more lateral veins per side in leaf, shape of spathe canoe, sessile spadix, and cathaphylls $3-5$.

The new species is also very closely related to Aglaonema hookerianum by having spadix sessile, terete and shorter than spathe, non-variegated ovate to elliptic lamina. But, differs by having erect but much shorter decumbent stem, $1-2$ inflorescences (usually single) at a time, curved spathe apex, female part not adnate to spathe, less leaf number (1 - 4 max.), not crowded at stem apex, and shorter peduncle (max. $5 \mathrm{~cm}$ long), thick terete spadix, ovate ovary. The major differences between these four species are provided in Table 1.

Table 1. Comparison of diagnostic morphological characters of Aglaonema tassai sp. nov. with its three related species

\begin{tabular}{|c|c|c|c|c|}
\hline Characters & A. hookerianum Schott & $\begin{array}{l}\text { A. simplex (Blume) } \\
\text { Blume }\end{array}$ & $\begin{array}{l}\text { A. modestum Schott. } \\
\text { ex Engl. }\end{array}$ & A. tassai sp. nov. \\
\hline Stem & $\begin{array}{l}\text { Erect, } 40-50 \mathrm{~cm} \text { tall, } \\
1.5-2.0 \mathrm{~cm} \text { diameter; } \\
\text { internodes } 1.5-3 \mathrm{~cm} \\
\text { long; rooting at nodes }\end{array}$ & $\begin{array}{l}\text { Erect, cylindric, } 40-80 \\
\mathrm{~cm} \text { tall, } 1-2 \mathrm{~cm} \\
\text { diameter; rooting at } \\
\text { nodes; internodes } 2-3 \\
\mathrm{~cm} \text { long }\end{array}$ & $\begin{array}{l}\text { Erect, stem green, } 40- \\
70 \mathrm{~cm} \text { tall, } 1.5-2 \mathrm{~cm} \text { in } \\
\text { diameter; internodes } \\
0.4-3 \mathrm{~cm} \text { long; } \\
\text { rooting at nodes }\end{array}$ & $\begin{array}{l}\text { Erect but lower part } \\
\text { decumbent, } 21-40 \mathrm{~cm} \\
\text { tall, } 1.5-2.6 \mathrm{~cm} \text { diameter, } \\
\text { terete, internodes } 1-2.0 \\
\mathrm{~cm} \text { long, rooting from } \\
\text { upper part of internodes } \\
\text { and at nodes }\end{array}$ \\
\hline
\end{tabular}


Hui Tag \& Atek Nangkar 311
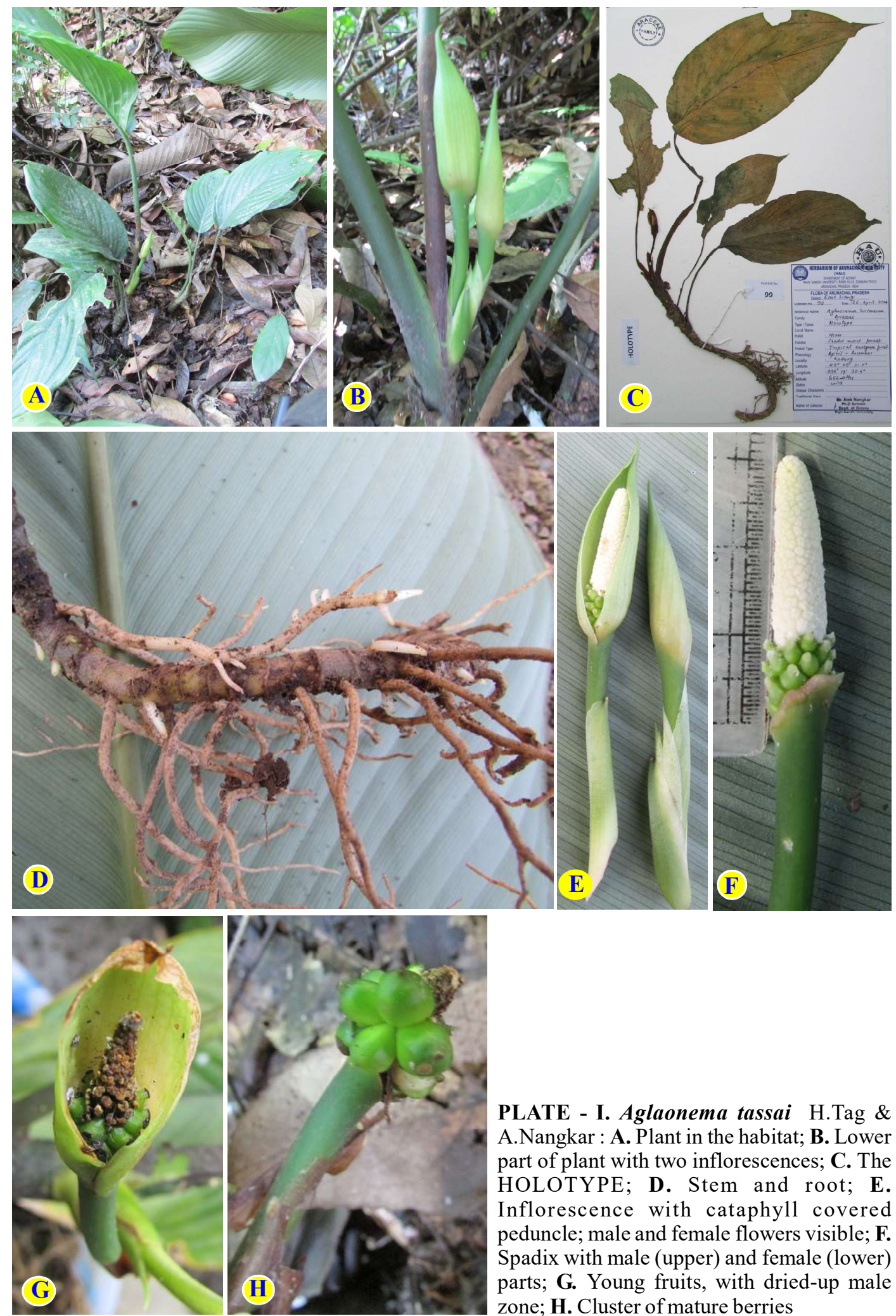

PLATE - I. Aglaonema tassai H.Tag \& A.Nangkar: A. Plant in the habitat; B. Lower part of plant with two inflorescences; $\mathbf{C}$. The HOLOTYPE; D. Stem and root; E. Inflorescence with cataphyll covered peduncle; male and female flowers visible; F. Spadix with male (upper) and female (lower) parts; G. Young fruits, with dried-up male zone; H. Cluster of mature berries 


\begin{tabular}{|c|c|c|c|c|}
\hline Characters & A. hookerianum Schott & $\begin{array}{l}\text { A. simplex (Blume) } \\
\text { Blume }\end{array}$ & $\begin{array}{l}\text { A. modestum Schott. } \\
\text { ex Engl. }\end{array}$ & A. tassai sp. nov. \\
\hline Lamina & $\begin{array}{l}\text { Leaves } 2 \text {, ovate to } \\
\text { elliptic or lanceolate to } \\
\text { narrowly elliptic, } \\
\text { apiculate - acuminate to } \\
\text { long acuminate, } 7-13 \\
\text { primary lateral veins; } \\
\text { petiole } 14-24 \mathrm{~cm} \text { long, } \\
0.7-0.9 \mathrm{~cm} \text { in diameter, } \\
\text { sheathing at base }\end{array}$ & $\begin{array}{l}\text { Leaves } 5 \text { or } 6 \text {, densely } \\
\text { crowded at stem apex, } \\
\text { ovate-oblong, caudate- } \\
\text { acuminate or long } \\
\text { acuminate, primary } \\
\text { lateral veins } 6-8 \text { per } \\
\text { side; petioles green, } 6 \text { - } \\
15 \mathrm{~cm} \text {, proximally } \\
\text { sheathing }\end{array}$ & $\begin{array}{l}\text { Leaves } 6, \text { mostly } \\
\text { crowded at stem apex, } \\
\text { ovate to ovate- } \\
\text { lanceolate, long } \\
\text { acuminate, primary } \\
\text { lateral veins } 4 \text { or } 5 \text { per } \\
\text { side; petioles green, } 5- \\
20 \mathrm{~cm} \text {, sheathing for } \\
\text { more than } 1 / 2 \text { of length }\end{array}$ & $\begin{array}{l}\text { Leaves } 2-4 \text {, not crowded } \\
\text { at stem-apex, elliptic to } \\
\text { ovate-oblong, caudate- } \\
\text { acuminate, primary lateral } \\
\text { veins } 6-20 \text { each side; } \\
\text { petioles } 13-18 \mathrm{~cm} \text { long, } \\
1.0-2.0 \mathrm{~cm} \text { in diameter, } \\
\text { sheathing at base }\end{array}$ \\
\hline Peduncle & $\begin{array}{l}1-3 \text { together, } 10-21 \\
\mathrm{~cm} \text { long. } 0.4 \mathrm{~cm} \text { in } \\
\text { diameter }\end{array}$ & $2-4,2-6 \mathrm{~cm}$ long & $\begin{array}{l}3 \text { together, } 10-12.5 \\
\text { cm long }\end{array}$ & $\begin{array}{l}1-2 \text { together, } 5-5.8 \mathrm{~cm} \\
\text { long, } 0.5 \mathrm{~cm} \text { in diameter }\end{array}$ \\
\hline Cataphyll & Absent & $\begin{array}{l}\text { Early caducous, broadly } \\
\text { linear, } 4-7 \mathrm{~cm} \text {, abruptly } \\
\text { acute }\end{array}$ & Absent & $\begin{array}{l}\text { Oblong, lanceolate, } 3-4 \\
\mathrm{~cm} \text { long, covering over } \\
\text { half the length of } \\
\text { peduncle, obtuse to acute }\end{array}$ \\
\hline Spathe & $\begin{array}{l}3.7-6 \mathrm{~cm} \text { long, } \\
\text { decurrent for } \\
(0.6) 1-1.5(2) \mathrm{cm} \text {, } \\
\text { stipe none; whitish green }\end{array}$ & $\begin{array}{l}\text { Cymbiform, ovoid, } 3- \\
4.5 \mathrm{~cm} \text { long, } 1.3 \mathrm{~cm} \text { in } \\
\text { diameter; light green }\end{array}$ & $\begin{array}{l}\text { Green, oblong- } \\
\text { lanceolate, } 5.5-7 \mathrm{~cm} \\
\text { long, } 1.5-2 \mathrm{~cm} \text { in } \\
\text { diameter, long } \\
\text { acuminate }\end{array}$ & $\begin{array}{l}\text { Spathe light green both } \\
\text { side, narrowly canoe- } \\
\text { shaped, } 4.5 \mathrm{~cm} \text { long, } 2.5 \\
\mathrm{~cm} \text { in diameter, shortly } \\
\text { acuminate, curved, middle } \\
\text { part open, semi-persistent, } \\
\text { macrescent, till fruits ripe }\end{array}$ \\
\hline Spadix & $\begin{array}{l}\text { Sessile, thin, cylindric, } \\
2.5-4 \mathrm{~cm} \text { long, } 0.8 \mathrm{~cm} \\
\text { short of spathe apex }\end{array}$ & $\begin{array}{l}2.5-4.5 \mathrm{~cm} \text { long, } \\
\text { slightly longer than or } \\
\text { equal to spathe, stipitate }\end{array}$ & $\begin{array}{l}\text { Thin cylindric, } 2.8 \mathrm{~cm} \\
\text { long, stipitate, shorter } \\
\text { than spathe apex }\end{array}$ & $\begin{array}{l}3.1 \mathrm{~cm} \text { long, sessile, } \\
\text { terete, somewhat conic, } \\
\text { shorter than spathe, } 1.2 \\
\mathrm{~cm} \text { short of spathe apex }\end{array}$ \\
\hline $\begin{array}{l}\text { Pistillate } \\
\text { zone }\end{array}$ & $\begin{array}{l}0.3-0.6 \mathrm{~cm} \text { long, major } \\
\text { part of pistillate portion } \\
\text { adnate to spathe; ovary } \\
\text { ovoid }\end{array}$ & $\begin{array}{l}0.5 \mathrm{~cm} \text { long, ovary } \\
\text { globose }\end{array}$ & $\begin{array}{l}0.5-0.8 \mathrm{~cm} \text { long, } \\
\text { ovary subglobose, } \\
\text { adenate to spathe }\end{array}$ & $\begin{array}{l}\text { Green, narrowly cylindric, } \\
0.7 \mathrm{~cm}-1 \mathrm{~cm} \text { long, } 0.8 \\
\mathrm{~cm} \text { in diam., ovary ovate, } \\
\text { pistillate part free, not } \\
\text { adnate to spathe }\end{array}$ \\
\hline $\begin{array}{l}\text { Staminate } \\
\text { zone }\end{array}$ & $\begin{array}{l}2-3.7 \mathrm{~cm} \text { long, } 0.3- \\
0.6 \mathrm{~cm} \text { thick, terrete, } \\
\text { slightly conic }\end{array}$ & $\begin{array}{l}2-3 \mathrm{~cm} \text { long, } 0.5 \mathrm{~cm} \\
\text { thick, conic }\end{array}$ & $\begin{array}{l}2-3 \mathrm{~cm} \text { long, } \\
\text { elongate, } 0.3-0.4 \mathrm{~cm} \\
\text { in diameter }\end{array}$ & $\begin{array}{l}2.1 \mathrm{~cm} \text { long, } 0.6 \mathrm{~cm} \text { in } \\
\text { diameter, terete, tip } \\
\text { rounded }\end{array}$ \\
\hline $\begin{array}{l}\text { Fruits and } \\
\text { Seeds }\end{array}$ & $\begin{array}{l}\text { Ripe berry red, } 2-3 \mathrm{~cm} \\
\text { long, } 0.9-1.4 \mathrm{~cm} \text { in } \\
\text { diameter; seed size not } \\
\text { known }\end{array}$ & $\begin{array}{l}\text { Ripe berry oblong, red, } \\
1.2-1.8 \mathrm{~cm} \text { long, } 0.7- \\
1.0 \mathrm{~cm} \text { in diameter; ovary } \\
\text { globose; seeds oblong, } \\
1.1-1.5 \mathrm{~cm}\end{array}$ & $\begin{array}{l}\text { Ripe berry oblong, } \\
\text { orange, } 2-3 \mathrm{~cm} \text { long, } \\
1.0-1.3 \mathrm{~cm} \text { in diameter; } \\
\text { ovary subglobose; seeds } \\
\text { oblong }\end{array}$ & $\begin{array}{l}\text { Ripe berry ovoid, red, } 0.4 \\
-0.6 \mathrm{~cm} \text { long and } 0.3 \mathrm{~cm} \\
\text { in diameter; ovary } \\
\text { unilocular; seed } 1, \\
\text { subglobose }\end{array}$ \\
\hline
\end{tabular}

Phenology: Flowering: April to June; Fruit ripening: November - December. Time of appearance: Seed germination observed during $1^{\text {st }}$ week of April to $3^{\text {rd }}$ week of May in tropical region.

\section{Key to species:}

1a. Stipe in spathe present 2

1b. Stipe in spathe absent

2a. $2.5 \mathrm{~cm}$ long spadix, slightly longer than or equal to spathe ............... A. simplex

2b. $2.8 \mathrm{~cm}$ long spadix, shorter than spathe apex

A. modestum

3a. Cylindric, $2.5-4 \mathrm{~cm}$ long spadix, $0.8 \mathrm{~cm}$ short of spathe apex..... A. hookerianum

3b. Terete, $3.1 \mathrm{~cm}$ long, $1.2 \mathrm{~cm}$ short of spathe apex A. tassai

TYPES: India: Arunachal Pradesh, East Siang District, Rottung Forest, latitude: $28^{\circ} 08^{\prime}$ 51.7" N; longitude: 95 14' 32.9"E; altitude: 656 m AMSL; Hui Tag \& Atek Nangkar 99, 


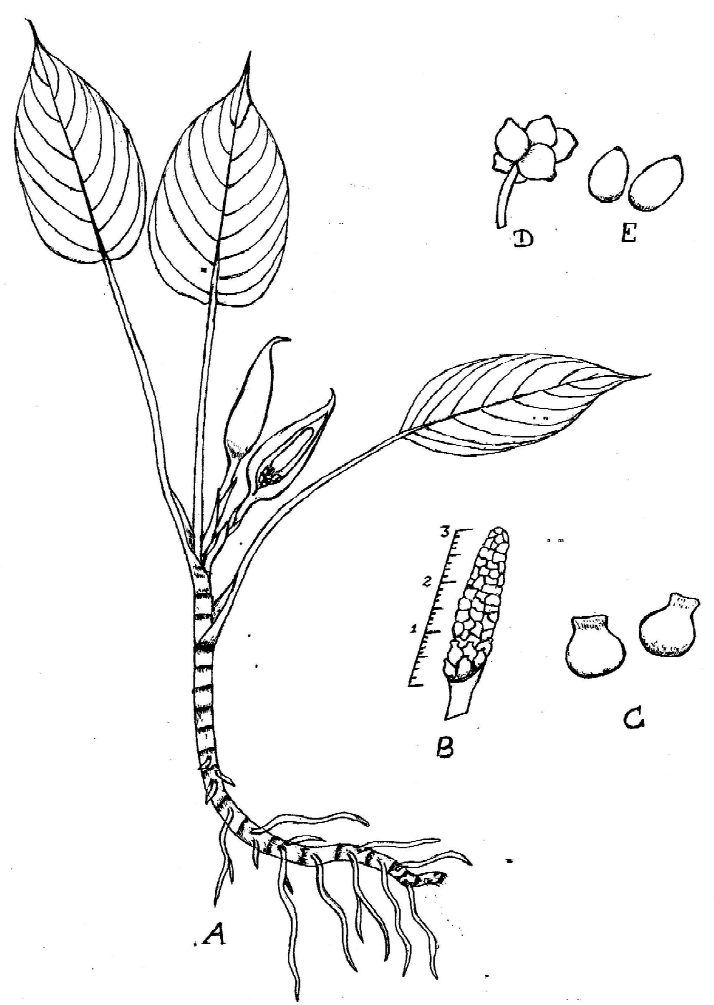

Figure 1. Labelled diagram of Aglaonema tassai Hui Tag \& Atek Nangkar: A. Whole plant; B. Spadix showing male and female zones; C. Female flowers; D. Cluster of fruits; E. Fruits dated 26.04.2014 (HOLOTYPUS: ASSAM)]; Hui Tag \& Atek Nangkar 100; dated 26.04.2014 (PARATYPUS: HAU)].

Habitat: Grows in dense moist evergreen forest floor, on shaded hills slope with altitudes of 400 - $700 \mathrm{~m}$ AMSL.

Conservation status: Known only from East Siang district of Arunachal Pradesh. Following IUCN (2001), criteria this taxon should therefore be treated as Data Deficient. The recorded natural habitat is not part of any type of Protected Area for biodiversity.

Etymology: The specific epithet 'tassai' is established in memory of the father of the first author (HT), Late Hui Tassa, from the domicile village New Gaa in the Kamle district of Arunachal Pradesh, India.

Distribution: So far, known only from one locality in the East Siang District of Arunachal Pradesh and is treated here as an endemic species.

\section{Acknowledgments}

The second author (AN) is thankful to the UGC, New Delhi, for awarding National Fellowship for ST \& SC students for pursuing Ph.D. Research. The authors are thankful to the UGC New Delhi for financially supporting the field work from CPEB-II programme. Authors deeply acknowledges the technical help rendered by Prof. A.P. Das on nomenclature and identity of the plant.

\section{LITERATURE CITED}

Blume, C.L.V. 1837. Aglaonema simplex (Blume) Blume. Rumphia 1: 152.

Engler, H.G.A. 1879. Aglaonema modestum Schott ex Engl. in A.L.P.de Candolle \& A.C.P.de Candolle, Monogr. Phan. 2: 442.

Hay, A. 1998. A new species of Aglaonema Schott (Araceae) from Terrenganu, Malayasia. Gard. Bull. Singap. 50: $1-4$.

Hooker, J.D. 1893. Aglaonema clarkei Hook.f. in The Flora of British India. Vol. 6. L. Reeve \& Co., 5 Henrieta Street, Covent Garden, London. Pp. 529.

IUCN 2001. IUCN Red List Categories and Criteria: Version 3.1. IUCN Species Survival Commission. IUCN, Gland, Switzerland and Cambridge, U.K.

Karthikeyan, S; Jain, S.K.; Nayar, M.P; Sanjappa, M. 1989. Florae Indicae Enumeratio: Monocotyledonae, Botanical Survey of India, Calcutta. Pp. 7 - 9. 
314 Aglaonema tassai sp. nov. from Arunachal Pradesh

Li, H \& Boyce, P.C. 2010. Aglaonema in Flora of China 23: 22-23.

Nicolson, D. H. 1969. A Revision of the Genus Aglaonema (Araceae). Smithsonian Contributions to Botany, No.1: 1 - 66. Smithsonian Institution Press, City of Washington, Washington DC, USA.

Schott, H.W. 1829. Aglaonema Schott, in Wiener Z. Kunst 892.

Schott, H.W. 1859. Aglaonema hookerianum Schott in Bonplandia (Hannover) 7: 30.

www.theplantlist.org [version 1.3, 2013] 